\title{
La cérémonie du thé dans la peinture anglaise du dix-huitième siècle
}

The Tea-Party in Eighteenth-Century British Painting

\section{Sophie Loussouarn}

\section{CpenEdition}

\section{Journals}

Édition électronique

URL : http://journals.openedition.org/rfcb/1637

DOI : $10.4000 /$ rfcb. 1637

ISSN : 2429-4373

Éditeur

CRECIB - Centre de recherche et d'études en civilisation britannique

Édition imprimée

Date de publication : 1 janvier 2006

ISSN : 0248-9015

Référence électronique

Sophie Loussouarn, "La cérémonie du thé dans la peinture anglaise du dix-huitième siècle ", Revue Française de Civilisation Britannique [En ligne], XIII-4 | 2006, mis en ligne le 01 janvier 2006, consulté le 19 avril 2019. URL : http://journals.openedition.org/rfcb/1637 ; DOI : 10.4000/rfcb.1637

Ce document a été généré automatiquement le 19 avril 2019

\section{(c) $(1)$}

Revue française de civilisation britannique est mis à disposition selon les termes de la licence Creative Commons Attribution - Pas d'Utilisation Commerciale - Pas de Modification 4.0 International. 


\title{
La cérémonie du thé dans la peinture anglaise du dix-huitième siècle
}

The Tea-Party in Eighteenth-Century British Painting

\author{
Sophie Loussouarn
}

1 Parler de la cérémonie du thé dans la peinture anglaise au dix-huitième siècle atteste des changements de goûts et de modes Outre-Manche et souligne le renforcement des particularités nationales. Les voyages en Asie, surtout en Chine et en Inde, les échanges commerciaux et le développement de l'East India Company expliquent l'introduction du thé comme boisson nationale au dix-huitième siècle. L'urbanisation, liée au développement des transports et du commerce, et l'évolution du niveau de vie suscitent le développement d'une société de consommation élargie, caractérisée par l'augmentation des achats domestiques et le renouvellement des articles de représentation sociale. Les élites qui disposent du loisir et des moyens nécessaires consomment de nouveaux produits de luxe qui soulignent leur appartenance sociale. Elles inventent de nouvelles formes de sociabilité et codifient les pratiques, avant que celles-ci ne se diffusent plus largement dans la société. La journée est rythmée par tout un cérémonial, commençant par le breakfast à 9 ou 10 heures du matin, puis le dinner à 2 ou 3 heures de l'après-midi. Depuis le début du dix-huitième siècle, après le dîner, les invités se retirent pendant une heure au salon pour prendre le thé et le dessert, jouer aux cartes ou écouter de la musique ${ }^{1}$. Dans la deuxième moitié du dix-huitième siècle, le dinner n'est servi qu'à 6 ou 7 heures du soir et entre le breakfast et le dinner se développe le rituel du thé.

2 Anna, la septième duchesse de Bedford, aurait été l'initiatrice de la cérémonie du thé entre quatre et cinq heures. La cérémonie devient de plus en plus somptueuse. Ainsi que le montrent les tableaux de l'époque, le thé est servi en famille dans le salon d'une demeure privée, ou en public, dans des jardins, tels Vauxhall ou Ranelagh, Bagnigge Wells et White Conduit House, ou dans des assembly rooms comme Carlisle House. La cérémonie du thé fait partie intégrante du raffinement des mœurs nationales. Elle contribue à 
écarter les hommes de l'alcool. Elle n'est plus le privilège de la noblesse, mais se répand chez les commerçants qui en assurent l'importation d'Inde par bateaux. Le thé nécessite des accessoires : des tasses et des théières en porcelaine, des bouilloires et des urnes en argent, qui symbolisent le statut social des consommateurs de thé. C'est dans la première moitié du dix-huitième siècle que les peintres Hogarth, Charles Phillips, Gawen Hamilton, Marcellus Laroon the Younger et Francis Hayman représentent dans leurs conversation pieces des familles qui prennent le thé et les petits gâteaux dans leur salon. Dans la deuxième moitié du dix-huitième siècle, le genre de la cérémonie du thé évolue. Johann Zoffany est le grand peintre qui représente la dimension intime de ce rituel dans ses conversation pieces. J.R Smith, quant à lui, se consacre au rite du thé dans la sphère publique, dans le tea-garden de Bagnigge Wells ou à Carlisle House, afin de souligner l'engouement du temps. À la fin du siècle, Rowlandson, James Gillray et Bunbury tournent en dérision la cérémonie du thé dans leurs caricatures qui dénoncent la vanité de la mode. La représentation du thé se transforme au fil du siècle et traduit l'évolution des rapports familiaux et l'évolution dans une certaine société.

\section{L'introduction du thé en Angleterre}

3 Le thé est introduit en Angleterre au dix-septième siècle en même temps que le chocolat et le café. Le thé arrive d'abord en petites quantités, grâce aux marchands portugais, puis grâce à l'East India Company, créée par charte royale, le 31 décembre 1600. Cette société, qui, au départ, a le monopole du commerce, devient un agent de l'impérialisme britannique en Inde, favorise le commerce du thé et des épices à partir de 1660, et importe des cargaisons de porcelaines, de soie et de tapis dans de grands bateaux. Le thé vient alors de Bantam, de Macao, de Surat et de Bombay. Mais c'est en fait Catherine de Bragance qui introduit la mode du thé à la cour d'Angleterre, lorsqu'elle épouse Charles II en $1662^{2}$. Au même moment, Thomas Garraway, le propriétaire d'une coffee-house vante les mérites du thé et en vend dans son établissement au cours de la deuxième moitié du dix-septième siècle. C'est à partir de ce moment que le thé devient une boisson prisée en Angleterre. Mais une livre de thé se vend 10 livres en 1657. Pepys lui-même consomme du thé et en vante les bienfaits dans ses mémoires : 'Mr Pelling the potticary tells (my wife) it is good for her cold and defluxion'.

4 L'East India Company joue un rôle prépondérant dans la diffusion du thé en Angleterre. La société rapporte deux variétés de thé : le thé noir qui a déjà fermenté et le thé vert qui n'est pas fermenté. Elle achète le thé en Chine pour un à deux shillings la livre et organise des ventes aux enchères dès 1677 . Les tea cutters (des voiliers cargos très effilés) rivalisent pour la gloire et le profit en apportant de Chine les premiers thés à chaque saison. En 1692, le gouvernment anglais abaisse l'impôt sur le thé à un shilling par livre. En 1706, Thomas Twining s'établit comme marchand dans le Strand à Londres où il vend du thé, du café, du chocolat, du brandy et de la noix de coco et un an plus tard, en 1707, Fortnum \& Mason, fondé par William, valet de pied de la reine Anne et par Hugh Mason, se spécialise dans le commerce des produits de luxe. À trente et un ans, Thomas Twining achète Tom's Coffee House, qui est parfaitement situé entre Westminster et la City de Londres et il devient le fournisseur de la noblesse et de la gentry. Parallèlement, il est employé par Thomas d'Aeth, riche marchand de l'East India Company. De nombreuses boutiques tenues par des femmes vendent du thé, notamment Mary Tuke, orpheline de trente ans qui se lance dans le commerce à York. Les marchands se rencontrent dans des centres 
d'échange, notamment le Royal Exchange et le Hall of Commerce dans Threadneedle Street, où s'effectuent des transactions et des ventes de thé aux enchères ${ }^{3}$.

Le thé reste cependant un luxe réservé à une élite, puisqu'en juin 1703, Sarah, duchesse de Marlborough, Mistress of the Robes de la reine Anne, achète du thé pour un souverain la livre. C'est une denrée si précieuse qu'on le conserve dans des coffrets en argent fermés à clé (tea caddy). Malgré son coût, le thé devient un produit de consommation à la mode dès 1710. Puis les réformes de Walpole vont contribuer à abaisser les prix de moitié dans la deuxième partie du dix-huitième siècle à 10 shillings la livre pour du thé noir et 12 shillings pour du thé vert ${ }^{4}$. Mais le thé fait l'objet de nombreuses attaques. Dès 1722 un pamphlet anonyme, Essay on the Nature, Use and Abuse of Tea in a Letter to a Lady, condamne le thé avec véhémence, le considère comme une conséquence du luxe que tolère l'ignorance et estime qu'il détruit l'orgueil, le courage et l'ambition'. En outre, dans Letter to a Friend Concerning Tea (1747), John Wesley incite son interlocuteur à abandonner le thé, qui est mauvais pour les nerfs et diminue la force physique. Enfin, dans son Essay on Tea (1756), Jonas Hanway condamne le thé, qui est nuisible aux enfants pauvres, et il estime que les sommes consacrées au thé seraient plus utilement employées à l'achat de lin ou de soie. Ce ne sont là que des voix isolées qui ne parviennent pas à ternir l'image du thé, vanté par John Coakley Lettsom dans son histoire du thé, The Natural History of the Tea-Tree with Observations on the Medical Qualities of Tea and Effects of Tea-Drinking:

So far therefore Tea, if not too fine, if not too hot, nor in too great quantities is perhaps preferable to any vegetable infusion we know. And if we take into consideration likewise its known enlivening energy it will appear that our attachment to Tea is not merely from its being costly or fashionable but from its superiority in taste and effects to most other vegetables. ${ }^{6}$

Les importations de thé en Angleterre ne cessent d'ailleurs d'augmenter au cours du dixhuitième siècle, passant de 66738 livres en 1701 à 890191 en 1741, 2774809 en 1751, 5566793 en 1771 et 15096840 en 1791. Il faut ajouter que la consommation du thé a entrainé la consommation de sucre, qui se développe grâce à l'essor du commerce avec les Antilles. En 1720, la consommation de sucre est de 8 livres par habitant par an et elle passe à 12 livres en 1770 .

\section{La cérémonie du thé: un nouveau genre pictural national}

Dès 1710, le thé devient un produit à la mode et la cérémonie du thé est représentée dans la peinture anglaise comme un rituel dès cette époque dans la gravure intitulée The TeaTable (c.1710), où deux messieurs regardent un groupe de femmes assises autour d'une table. À partir des années 1720 se développe la mode des conversation pieces qui représentent une famille ou un groupe d'amis, réunis de manière informelle, dans leur maison ou dans leur jardin. Ce type de peinture, importé de Hollande et de France, devient typiquement britannique et reflète l'évolution des comportements et des modes de représentation en Angleterre ${ }^{7}$. Le souci de se conformer au code du paraître des élites conduit les familles à se faire représenter dans leur demeure, en train de prendre le thé, boisson préférée des femmes et loisir de prédilection en famille ou entre amis. Les femmes sont le centre de gravité de ces conversation pieces, tandis que les hommes et les domestiques en sont les satellites. En cela, les peintres montrent l'influence civilisatrice des femmes qui participent au raffinement des mœurs et à la bonne marche d'une 
maison. Les Anglaises jouent en effet un rôle grandissant dans la sphère domestique et influencent la décoration intérieure. Elles contribuent à créer une atmosphère intime qui s'éloigne du formalisme du passé ${ }^{8}$. Elles président à la cérémonie du thé, qui est accompagnée de conversation et de jeux de cartes, voire parfois de musique. La représentation de ce rituel varie d'un milieu à l'autre, d'une famille à l'autre, mais elle donne aux Anglais le sens de leur identité nationale. La cérémonie du thé met en jeu l'art de la table et de la décoration en Angleterre au dix-huitième siècle et incarne le statut social de la famille et de ses hôtes. Les tableaux de la cérémonie du thé montrent l'influence du style palladien sur l'architecture britannique. Les tableaux figurent souvent des plafonds peints, des colonnes, des frontons à l'antique, caractéristiques des goûts artistiques britanniques de l'époque et ils montrent des tableaux dans le tableau, signe de l'opulence de la famille. Ils incluent aussi souvent une cheminée surmontée de statues et des portes donnant de la profondeur au tableau et offrant une échappée sur le jardin, marque de l'identité sociale. En outre, ils révèlent les vertus classiques d'ordre, de symétrie et d'équilibre qu'admirent les élites britanniques.

8 La cérémonie du thé est conçue pour faciliter la conversation, qui se déroule souvent autour d'une table simple ou à rebords, en acajou ou en argent, à pieds droits ou cabriolet, sur laquelle est posée une théière en porcelaine rapportée dans les cargos de l'East India Company, avant 1740 , date à laquelle se développe la fabrication de théières dans le Staffordshire. Dans les années 1740 à 1760, les manufactures anglaises se mettent à imiter les modèles orientaux et les tableaux représentent les services à thé en porcelaine à pâte tendre, avec des décors floraux polychromes fabriqués à Chelsea, Bow, Worcester, Etruria (Wedgwood) et Derby. Enfin, certains tableaux font aussi figurer une immense bouilloire en argent, sur un trépied, avec un grand bec verseur et des poignées ou encore la boîte à thé en argent, souvent aux armes de la famille, qui symbolisent la dignité de la cérémonie. L'argenterie de l'époque s'inspire du style de Meissonier sous la Régence en France.

La représentation du rituel du thé varie selon qu'il s'agit de la cour, de la noblesse ou de la gentry. À la cour et dans la noblesse, le thé est toujours servi par des domestiques que le peintre inclut dans le tableau, car ils font partie de la maison et révèlent le pouvoir économique de la famille. Les deux peintres qui représentent la cérémonie du thé à la cour sont Charles Philips et Marcellus Laroon. Charles Philips (1708-1747), peintre de la famille royale et de la haute aristocratie, a adapté le style de la conversation piece de Hogarth et a réalisé une peinture achevée avec la Tea-Party at Lord Harrington's House (1730) ${ }^{9}$. Cette cérémonie du thé représente des courtisans en fonction de leur naissance et de leur rang : Lady Betty Germain, vêtue de bleu est assise à côté de la duchesse de Montagu, vêtue d'une robe de dentelle et coiffée d'un voile assorti à sa robe. En face de Lady Betty Germain, la duchesse de Dorset est vêtue de rose. Ce tableau se compose de trois groupes assis autour de trois petites tables: sur la gauche, deux domestiques regardent un groupe de cinq personnes qui prennent le thé, au centre, une servante observe quatre personnes qui jouent au whist et Lord Harrington relie les trois groupes. Il tient à la main un bâton et un chapeau. Deux lustres pendent au plafond et le mobilier souligne l'opulence du maitre de céans : deux peintures de fleurs au-dessus des portes, un paysage au-dessus de la cheminée, deux tapisseries bleues, à gauche et à droite, et un tapis oriental. La pose des personnages et la richesse de leurs costumes, l'insigne de l'ordre de la Jarretière que porte le personnage assis à droite, symbolisent aussi leur dignité et s'inscrivent dans une logique d'ostentation. 
10 Marcellus Laroon le Jeune (1679-1772) traite aussi de la cérémonie du thé à la cour dans deux tableaux : A Musical Tea Party, qui décrit une assembly à Kew Palace ${ }^{10}$, et A Musical Party, qui se tient chez le duc de Buccleugh. A Musical Tea Party est une peinture excessivement complexe à décoder, axée autour d'un portrait équestre à la Van Dyck. À gauche, quatre personnes sont groupées autour d'une table, tandis qu'à droite un couple assis de dos, deux personnages debout buvant du thé et un pasteur écoutent un claveciniste et un violoniste. Une servante verse de l'eau chaude dans la théière, un domestique apporte un plateau de fruits, un négrillon fait passer les tasses à thé et un autre domestique tire le rideau. Le mobilier somptueux (coffre en laque surmonté d'une porcelaine de Delft) contraste avec le mobilier simple de la gentry. Le thé est servi dans un beau service en porcelaine à motifs floraux avec un plateau de biscuits et de fruits. Tout comme dans le tableau de Charles Philips, les personnages sont en représentation dans une mise en scène quasi théâtrale et ils respectent l'étiquette dictée par leur appartenance sociale.

11 La cérémonie du thé dans la noblesse souligne aussi la vie fastueuse des pairs du royaume. Ces tableaux de la première moitié du dix-huitième siècle révèlent l'influence du style palladien en Angleterre sous le règne de Georges II. C'est à cette époque que les classes dirigeantes s'approprient le modèle de la Renaissance vénitienne pour le transformer en style national. Wanstead, la grande demeure de Castlemain et Wentworth, le château du comte de Strafford, en sont d'excellents exemples. Hogarth peint The Assembly at Wanstead House (1728-1731) ${ }^{11}$ pour Sir Richard Child, vicomte Castlemain (Earl Tilney), le riche propriétaire de Wanstead House dans l'Essex et son épouse Dorothy Glynne. Le vicomte est assis sur un siège en bois doré richement orné de volutes et de bulbes, devant une table en argent sur laquelle est posé un service à thé, à côté d'une cheminée en marbre surmontée d'un buste et d'une peinture et entourée de deux grandes fresques historiques. La pièce, somptueusement décorée dans le style de William Kent, avec un plafond en trompe l'œil et des frontons aux portes, regroupe une vingtaine d'invités du vicomte Castlemain après le dîner. Un domestique allume l'un des deux lustres. Tout comme chez Charles Philips, la peinture se décompose en plusieurs groupes, autour de deux tables de jeu et d'une table à thé. Les enfants du maître de maison, descendants de la lignée, sont regroupés à l'extrémité gauche du tableau. Dans le tableau The Earl of Strafford and his Family (1732) ${ }^{12}$, le portraitiste écossais Gawen Hamilton peint Thomas Wentworth (1672-1739), $1^{\text {er }}$ comte de Strafford et ancien ambassadeur à Berlin, avec son épouse Anne, fille et héritière de Sir Henry Johnson of Bradenham, son fils William (né en 1722) et ses trois filles Anne, Lucy et Henrietta dans le grand hall de Wentworth Castle. Le comte, décoré de l'Ordre de la Jarretière est assis sur un fauteuil surmonté d'une couronne comtale et il trône dans son entrée conçue par Jean Bodt en 1708. L'escalier éclairé par les lustres donne de la profondeur au tableau et ouvre sur un plafond peint et une galerie, au premier étage. L'architecture symbolise le prestige social de la famille. Des pilastres soutiennent les poutres et le blason du comte est représenté au-dessus de la voûte qui mène au grand escalier ${ }^{13}$.

12 Les membres de la chambre des Communes se font aussi peindre à l'occasion de la cérémonie du thé. Arthur Devis peint la famille d'un député dans The Crewe Conversation Piece (1743-44). John Crewe, député du Cheshire de 1734 à 1752 et fervent conservateur se tient à gauche du portrait, appuyé sur le dos de la chaise de son épouse. Sa fille tient la canne à pommeau d'or de son père d'une main et un biscuit d'une autre. Le pasteur, qui incarne l'ordre social, est adossé sur le fauteuil du père de John Crewe, assis à côté de 
deux dames. Le frère de John Crewe tient dans ses bras un bébé. Le salon est éclairé par les fenêtres palladiennes qui ouvrent sur le jardin et le temple classique. L'architecture de la maison, les frises, les plafonds ainsi que le tableau de ruines antiques, au-dessus de la porte à droite, manifestent le pouvoir politique et la culture classique de cette famille de notables. Le portrait de la cérémonie du thé devient une légitimation de la puissance de la famille. The Strode Family (c. 1738) ${ }^{14}$ de Hogarth (Hors-texte 1) représente autour de la table William Strode de Ponsbourne Hall, Hertfordshire, et député de Reading, son épouse, Lady Anne Strode (1711-1752), fille du $5^{\mathrm{e}}$ comte de Salisbury, et son frère, le colonel Samuel Strode, qui ont invité un ami, le Dr Arthur Smyth (1707-1771), plus tard archevêque de Dublin, qui tient un livre à la main. L'intendant des Strode, Jonathan Powell, verse de l'eau dans une théière placée sur un plateau en argent sur une table ronde. La décoration de la pièce symbolise l'appartenance sociale de la famille avec un plafond peint, un grand paysage à droite et deux petits cappricios dessous. À l'arrière-plan, on devine une bibliothèque qui révèle l'intérêt des Strode pour la culture. Le tableau est encadré à gauche d'un rideau et à droite d'une porte.

Hors-texte 1 : Hogarth, The Strode Family (c.1738)

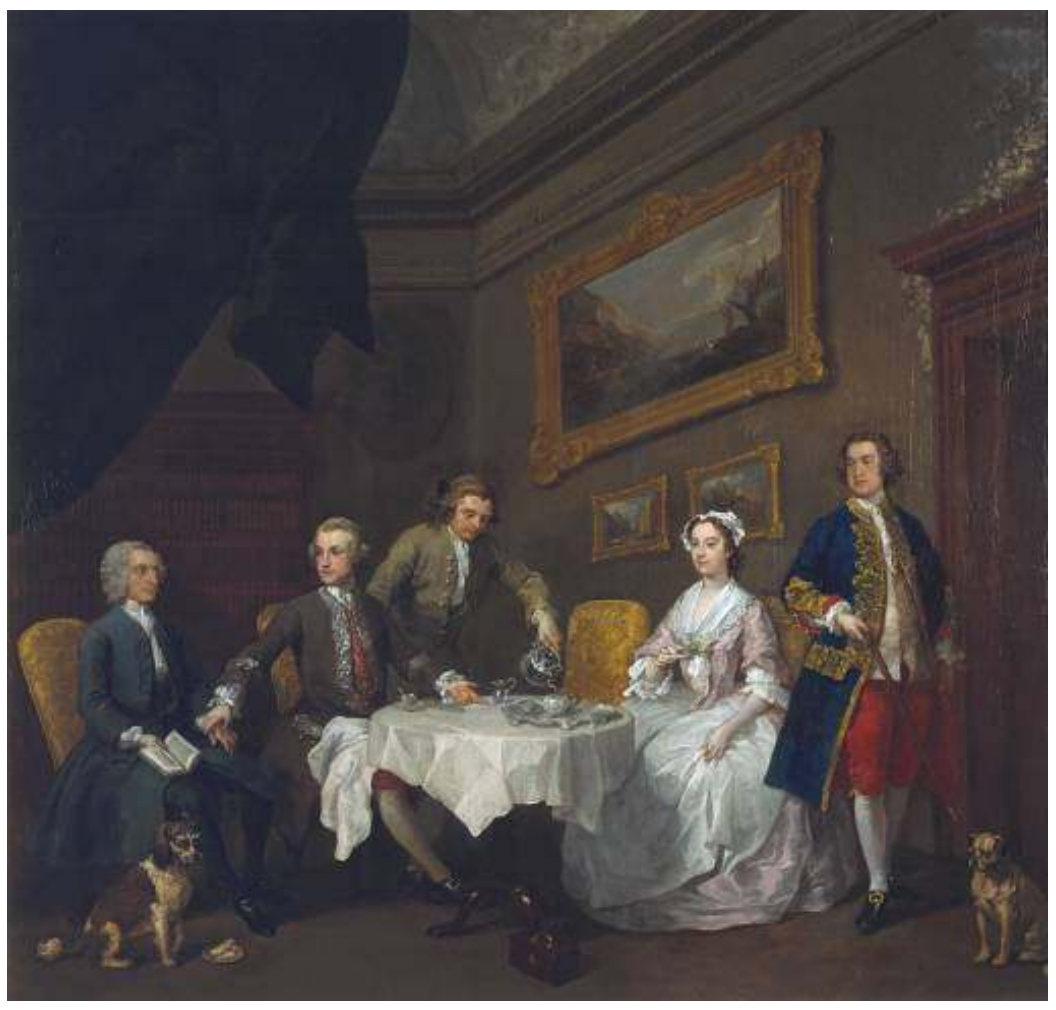

Les autres tableaux de Hogarth et de Gawen Hamilton représentent la cérémonie du thé dans des familles de la gentry: les Wollaston, les Strong et les Western. The Wollaston Family (1730) de Hogarth ${ }^{15}$ souligne la richesse de William Wollaston, propriétaire de Finborough Hall dans le Suffolk, qui réunit le cercle étroit des amis de la maison pour prendre le thé et jouer aux cartes après le dîner. La décoration du salon avec les colonnes corinthiennes, le lustre en argent, les chaises et la table à thé en argent soulignent le statut social du maître de maison, qui relie les deux groupes en un tout. Le regard de l'hôte dans la direction de son épouse met en relief les sentiments qu'ils éprouvent l'un pour l'autre et l'affection qui unit tous les personnages regroupés dans ce salon pour leur plaisir. 
D'autres tableaux nous font pénétrer dans l'univers plus simple des classes moyennes. Les portraits de Francis Hayman (1708-1776), Jonathan Tyers with his Family (1740) ${ }^{16}$ et The Gascoigne Family (c. 1740) ${ }^{17}$, ont une facture identique. Le peintre représente ces deux familles nucléaires dans un salon à la décoration sobre où une cheminée semblable à celle de James Gibbs se situe au centre du tableau, derrière la table à thé autour de laquelle sont assis le maître et la maitresse de maison entourés de leurs enfants. Un paravent donne de la profondeur au tableau qui figure une scène de la vie quotidienne, la cérémonie du thé, et met en jeu des conventions sociales et artistiques. À la différence des tableaux qui représentent de somptueuses pièces de réception, ces portraits font apparaître des espaces à caractère privé, des boudoirs, garnis de tentures et de paravents. Dans le tableau des Gascoigne, le service à thé en porcelaine bleue et blanche est importé de Chine. La théière hexagonale et le tapis turc sont des accessoires qu'utilise Hayman dans ces deux tableaux. Le tableau de William Verelst, The Gough Family (1741) ${ }^{18}$, d'inspiration flamande, est caractéristique du goût des années 1740 et représente cette famille de marchands annoblis dans son salon à Edgbaston. La mère et la fille sont de chaque côté de la table où sont posés les six tasses à thé, la théière et le tea caddy aux armes de Sir Henry, créé baronet en 1728. Avocat au Middle Temple et directeur de l'East India Company de 1735 à 1751, Sir Henry, interrompu dans ses écritures, est assis à son secrétaire, tandis que son fils fait le lien entre les deux groupes. Cette peinture qui représente la famille nucléaire est empreinte de simplicité, de bien-être et de confort, qui contrastent avec les réceptions mondaines à la cour et dans la noblesse.

\section{L'évolution d'un genre}

15 Alors que la cérémonie du thé dans la peinture des années 1730 représente une zone intermédiaire entre la sphère publique et l'intimité domestique, les images de la deuxième moitié du dix-huitième siècle s'attachent davantage à la représentation de la famille nucléaire et contrastent avec l'intimité superficielle des premiers tableaux ${ }^{19}$. Les intérieurs bourgeois et aristocratiques se transforment selon une logique de mise à distance des domestiques, perçus de plus en plus comme une présence gênante. Il convient aussi de remarquer que la cérémonie du thé n'est plus seulement représentée dans un salon comme dans Mr and Mrs Hill (c. 1750-51) d'Arthur Devis ${ }^{20}$ ou dans Lord Willoughby de Broke and his Family (1775) de Johann Zoffany (1733-1810) ${ }^{21}$, mais le thé se prend aussi dans le jardin de la propriété comme le montre Zoffany dans Mr \& Mrs Garrick at Tea entertaining Dr Johnson at their Villa at Chiswick ou The Auriol Family.

Le portrait de Johann Zoffany (1733-1810), Lord Willoughby de Broke and his Family (1775), peint pour John, $14^{\mathrm{e}}$ Baron Willoughby de Broke en 1771-2, est un témoignage de la vie privée de la noblesse au dix-huitième siècle et Zoffany a saisi avec beaucoup de naturel le charme de la vie familiale anglaise. Cette scène qui se déroule dans la breakfast room de Compton Verney, Northamptonshire, représente John et sa femme, Lady Louisa North (1737-98), sœur du Premier ministre, et leurs trois enfants: John (1762-1820), qui deviendra le $15^{\mathrm{e}}$ baron, George (1763-1773) et Louisa (1769-1835), qui épousera le Révérend Robert Barnard. Le père pointe un doigt réprobateur vers le petit enfant qui est debout à gauche de la table et se sert subrepticement un toast beurré. Le troisième enfant à droite traine son petit cheval de bois rouge. Le salon qui était un espace public pour les grandes familles devient un espace où les époux et leurs enfants mineurs, formant la famille, s'isolent du personnel. Dans cet espace clos se réunit la famille restreinte 
patriarcale où règne un sentiment d'affection qui unit les deux époux. Cette peinture crée une atmosphère d'intimité familiale dégagée des contraintes sociales. La cheminée surmontée d'un tableau, les bronzes, le tapis, la table, le service à thé en porcelaine de Chine, les reflets de l'urne à thé en argent et les jouets d'enfants expriment la chaleur du foyer. La composition de la peinture est admirable et les personnages prennent vie dans ce chef d'œuvre de Zoffany qui souligne le triomphe du sentimentalisme, l'évolution des rapports familiaux et l'intérêt que les parents prennent aux activités ludiques de leurs enfants. Ce tableau est loin du formalisme des conversation pieces de la première moitié du dix-huitième siècle, notamment An Assembly at Wanstead House (1728-31) de Hogarth ou The Earl of Strafford and his Family (1732) de Gawen Hamilton. D'autre part, les tableaux peints sous le règne de Georges III présentent des intérieurs néo-classiques qui contrastent avec les intérieurs de style palladien que peignaient les artistes du règne de Georges II. La mise en scène théâtrale qui met en valeur les décors du plafond, les tentures et le mobilier est remplacée par un cadrage naturel où ces personnages expriment leur humanité avec simplicité.

Le grand tableau de la famille Auriol, peint en Inde vers 1780, nous transporte sur les lieux de production du thé et révèle l'importance de l'Inde dans la politique coloniale et commerciale de la Grande-Bretagne. Zoffany représente trois groupes : l'un prend le thé, l'autre joue aux échecs, et le troisième s'adonne au plaisir de la conversation. Cela révèle l'influence du mode de vie de la métropole sur la colonie. Au centre, deux dames: Charlotte, devenue Mrs Thomas Dashwood, et Sophia, devenue Mrs John Prinsep, sont assises autour d'une table et prennent le thé, versé par un domestique noir. Le thé est servi dans une théière en argent et de la porcelaine. À leur gauche, Thomas Dashwood, le deuxième fils de Sir James Dashwood de Kirklington, Oxford, joue aux échecs avec un ami qui s'entretient simultanément avec deux indigènes en costume local. À gauche de la peinture se tiennent Charles Auriol et James Auriol, qui devisent avec John Prinsep. Derrière eux se tient un autre domestique.

Un autre tableau de plein air, Mr \& Mrs Garrick at Tea entertaining Dr Johnson at their Villa at Chiswick, présente la cérémonie du thé dans un cadre naturel empreint de liberté. Mr et Mrs Garrick et leur invité d'honneur le Dr Samuel Johnson sont assis au bord de la rivière et prennent le thé, avec un autre invité, Mr Bowden, qui se tient derrière la chaise de l'hôtesse, alors que le frère de Garrick, George Garrick, pêche à la ligne. Cette scène dénuée de conventions sociales est imprégnée de simplicité bucolique. L'atmosphère de sérénité est créée par la composition limitée à l'horizon par des arbres et des saules qui se reflètent dans l'eau. La présence d'une île amorce un style nouveau quasi romantique.

Si le thé n'est pas une boisson réservée aux femmes, il devient aussi la grande boisson servie au petit-déjeuner dans la noblesse comme dans la gentry, comme le montrent quelques rares peintres anglais de la fin du dix-huitième siècle. Ces tableaux soulignent l'évolution des goûts et du genre de la cérémonie du thé en peinture. Zoffany a peint un petit déjeuner de chasse où est servi le thé sur une nappe blanche dans de belles tasses en porcelaine dans son Group representing a Hunt Breakfast at Holme Park, Berkshire ${ }^{22}$. Cette peinture regroupe autour de Mr Robert Palmer, député de Holme Park, ses amis, le duc de Grafton, le duc de Bedford, allié politique du duc de Grafton, Sir Richard Aldworth of Stanlake Park, et ses trois gendres, Sir Thomas Beauchamp-Proctor of Langley Park, Mr Francis Pym, MP, et Mr George Beauchamp-Proctor. Cette peinture montre donc que les hommes boivent du thé au petit-déjeuner en Angleterre, alors qu'ils boivent du café en France. Enfin, dans The Breakfast Room at 12 Lincoln's Inn Fields (c. 1798), Joseph Michael 
Gandy représente l'architecte Soane qui lit son journal à côté de la cheminée, tandis que son épouse lui sert le thé au petit déjeuner à Soane's House, construite en 1792-93. Cette peinture intime représente simplement le couple dans son cadre quotidien sans aucune ostentation. Les murs de la pièce sont tapissés de livres, le plafond a des voûtes décorées de treillis, de chevrefeuille et d'ancolie, comme si la pièce était une véranda. Les gravures de Piranèse et les sculptures antiques révèlent les goûts de l'architecte collectionneur. L'intrusion de la nature et une telle simplicité témoignent d'un changement de mode dans la conversation piece de la fin du dix-huitième siècle qui contraste avec le rituel somptueux de la première moitié du siècle.

À la fin du dix-huitième siècle, apparaissent des jardins publics où il devient à la mode de prendre le thé. Outre les jardins de Vauxhall et Ranelagh, Bagnigge Wells et White Conduit House permettent à des Anglais de condition plus modeste de prendre le thé en plein air ou dans une salle. J.R. Smith peint ainsi Bagnigge Wells en 1772, qui tourne en dérision les courtisans et les dandys de l'époque. La peinture de George Morland (1763-1804), The Tea Garden ${ }^{23}$ correspond au développement des pleasure-gardens et représente un couple avec la mère qui tient son enfant, le fils qui sert le thé et les deux petits enfants qui jouent avec un chien et un cheval de bois. Ceci souligne l'importance du thé dans la vie familiale anglaise où se retrouvent toutes les générations regroupées autour de plaisirs simples.

21 Dans un tout autre style, Gillray désacralise la cérémonie du thé à la cour dans sa caricature de Georges III, de la reine Charlotte et des six princesses (Figure 1).

Figure 1 : Anti-Saccharites, - or John Bull and his Family Leaving off the use of Sugar (1792)

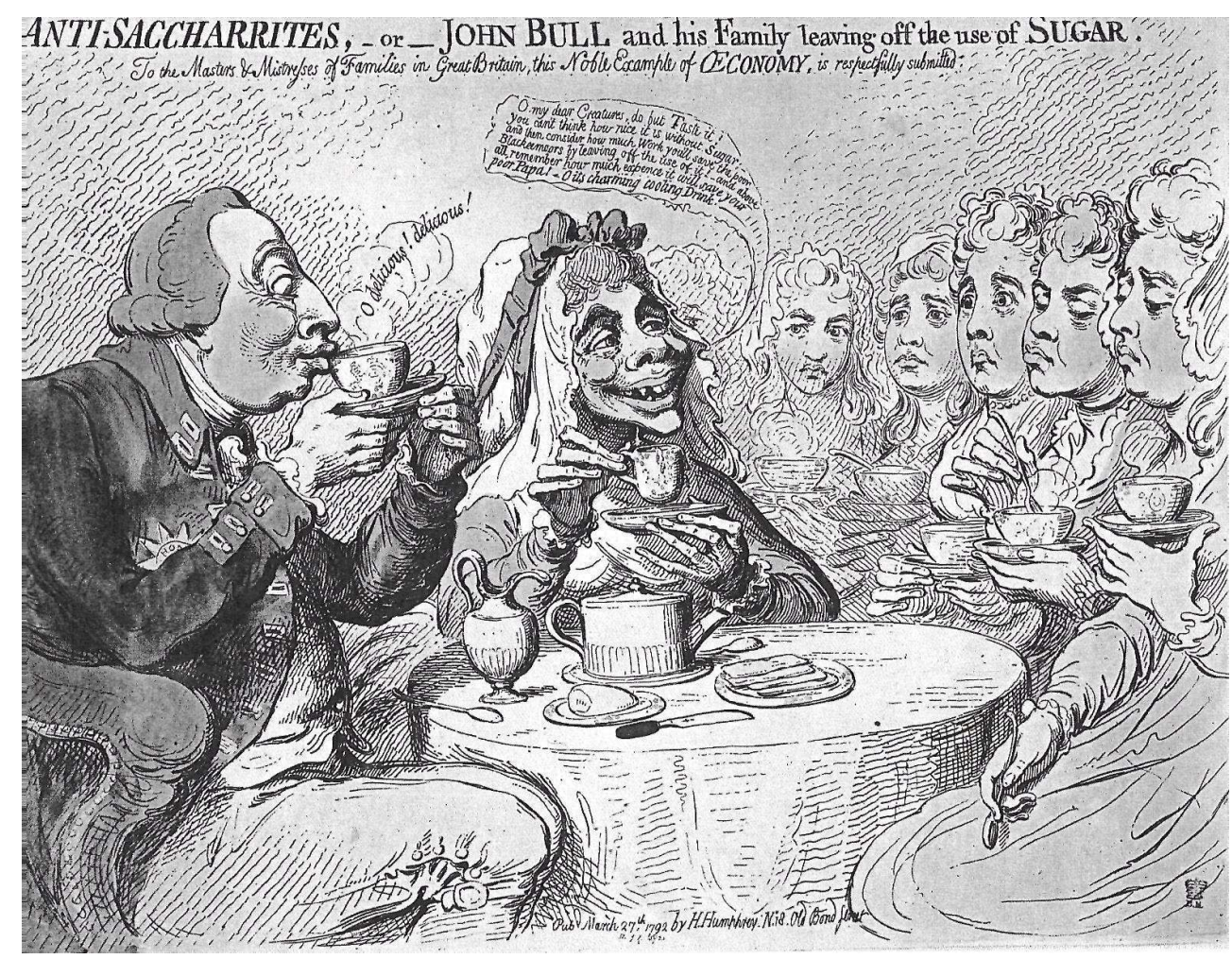

Le caricaturiste tourne en dérision la mesquinerie du roi et de la reine qui privent leurs filles de sucre dans leur thé par souci d'économie et pour donner l'exemple aux familles 
anglaises qui consomment des produits de luxe. L'avarice du couple royal a été mise en pièces par Gillray dans plusieurs caricatures. Ici la reine Charlotte s'exclame ainsi :

O my dear Creatures do but Taste it! You can't think how nice it is without Sugar: and then, consider how much work you'll save the poor blackeemoors by leaving off the use of it! - and above all, remember how much expence it will save your poor

Papa! - O its charming cooling drink.

La satire du statut du roi le ramène au niveau des classes moyennes parcimonieuses qui se mettent à boire du thé grâce à l'essor du commerce et à l'augmentation du niveau de vie. Un Français prisonnier à Londres met aussi en pièces la tradition du thé à l'anglaise, dans une gravure intitulée Les Dames anglaises après-diné (1814), gravée à Paris chez Martinet ${ }^{24}$. Le thé est représenté comme une boisson féminine, qui réunit toutes les générations, des personnes âgées aux enfants, en passant par les jeunes filles. Le peintre dénonce le comportement apprêté des femmes et insiste sur les difformités corporelles. De telles caricatures sont bien loin des conversation pieces visant à faire passer une famille à la postérité. A la fin du dix-huitième siècle, la satire devient une arme entre les mains du peintre, qui se veut moraliste et critique les extravagances de la mode anglaise.

\section{Conclusion}

Ces peintures de la cérémonie du thé témoignent d'un nouveau style de vie élitiste que les échanges commerciaux et la politesse des mœurs ont apporté à la nation anglaise. Elles sont le reflet de l'idéologie dominante selon laquelle le raffinement est le fruit de l'éducation et de la sociabilité. Elles se font également l'écho des théories de David Hume, qui estime que l'opulence et le raffinement sont intimement liés à l'essor du commerce, qui confère la sécurité et le bonheur aux individus. Selon lui, le luxe contribue au bonheur des hommes en les faisant bénéficier de nouveaux plaisirs ${ }^{25}$. La représentation picturale de la cérémonie du thé révèle le style de vie des hommes et des femmes policés appartenant à la noblesse ou à la gentry, mais aussi au monde des affaires ou de la politique, elle dévoile leurs costumes, leur mobilier, leurs peintures et leur argenterie, mais aussi leurs manières. Hogarth, Hayman ou Zoffany ont tous trois construit une image de ce trait de l'identité nationale, qui contraste avec les portraits des peintres étrangers. Ici, le luxe que symbolise la cérémonie du thé constitue un élément du prestige de la famille représentée dans son cadre de vie quotidien, le plus souvent dans un salon, mais aussi parfois en plein air. La cérémonie du thé est tantôt un rite relevant de la sphère publique, surtout dans la première moitié du dix-huitième siècle, tantôt un moment de l'intimité d'une famille nucléaire. La maîtresse de maison à côté du maitre de maison joue son rôle face aux domestiques et aux voisins. La grande variété de tableaux de la cérémonie du thé peints en Angleterre souligne combien cette tradition est devenue une institution quotidienne et exprime l'identité anglaise où la vie de la communauté nucléaire ou élargie au groupe s'oppose aux plaisirs frivoles souvent mis en scène dans la peinture française.

En France, en revanche, les peintres représentent la cérémonie du café ou du chocolat, qui sont devenus des boissons à la mode dès le dix-septième siècle. En effet, le café conquiert la haute société parisienne vers 1669, par l'intermédiaire de l'ambassadeur de l'empire ottoman à Paris, Soliman Aga. Des plants de café seront offerts à Louis XIV, qui les confiera aux botanistes du Jardin du roi. De là, le caféier sera introduit dans les colonies antillaises, où la France s'approvisionnera librement. Lancret peint une Famille 
dans un Jardin (1742), où parents et enfants prennent le café dans un jardin, à l'écart du monde et où la fillette goûte au breuvage pour la première fois ${ }^{26}$. Par ailleurs, le cacao, introduit en Martinique en 1660 par Marie-Thérèse d'Autriche, femme de Louis XIV, devient très à la mode à Versailles et dans les salons aristocratiques pour ses vertus digestives et ses propriétés dynamisantes. La consommation du chocolat augmente en France au dix-huitième siècle, mais reste l'apanage d'une minorité de nobles qui le sirotent à la cour et dans les salons. Jean-Baptiste-André Gautier-Dagoty (1740-1786) représente une famille réunie dans une bibliothèque en train de prendre le chocolat dans Portrait d'une Famille (1770) ${ }^{27}$. De même Jean Baptiste Charpentier le Vieux peint-il la famille du duc de Penthièvre en 1768 dans La Tasse de Chocolat (1768) ${ }^{28}$.

En Angleterre, le café et le chocolat ne supplanteront jamais le thé, qui devient la boisson nationale réservée à la noblesse et à la gentry. La représentation de la cérémonie du thé revêt donc un caractère national et témoigne d'un certain décorum, signe d'appartenance sociale et familiale. Ce genre de peinture devient un moyen de promouvoir la supériorité morale et culturelle de l'Angleterre, où la consommation du thé exprime une communauté de goûts.

\section{BIBLIOGRAPHIE}

CORNFORTH, John. Early Georgian Interiors. New Haven: Yale University Press, 2004.

GIROUARD, Mark. Life in the English Country House. New Haven: Yale University Press, 1978.

SMITH, Charles Saunarez. Eighteenth Century Decoration: Design and the Domestic Interior in England. London: Weidenfeld \& Nicolson, 1993.

SOLKIN, David H. Painting for Money : The Visual Arts and the Public Sphere in Eighteenth-Century

England. New Haven: Yale University Press, 1993.

WATERHOUSE, Ellis. Painting in Britain, 1530-1790. New Haven: Yale University Press, 1994.

\section{NOTES}

1. Mark GIROUARD analyse l'évolution de la sociabilité en Angleterre dans les demeures au chapitre 7 de Life in the English Country House, New Haven: Yale UP, 1978, pp. 191-205. Il fait même remonter la tradition du thé servi après le dîner ou le souper aux années 1670 et 1680 . Ce qui allait devenir une institution pour les classes supérieures britanniques commence comme un intermède au cours duquel les femmes préparent le thé ou le café, avant que les hommes ne viennent les rejoindre après avoir parlé de politique. John CORNFORTH mentionne également l'utilisation des salons par les femmes pour prendre le thé après le dîner, dans son livre Early Georgian Interiors, New Haven: Yale UP, 2004, p. 53.

2. Denys FORREST développe l'arrivée du thé en Angleterre dans son ouvrage Tea for the British : The Social and Economic History of a Famous Trade, London: Chatto \& Windus, 1973. 
3. Un guide de Londres, A New Survey of London fully developping its Antiquity, History and Architecture, London: John Weale, 1853, fait état du développement du commerce du thé et Rowlandson représente une vente aux enchères de thé à l'East India House.

4. Tel est le prix payé par le duc de Bedford qui achète son thé à Richard Haines à Tom's Coffee House.

5. L'auteur critique le the comme 'one of the many errors which are introduced by luxury, suffered through ignorance', London, 1722, p. 6, p. 39.

6. The Natural History of the Tea-Tree, London: Printed for Edward \& Charles Dilly, 1772, Part II, Section 5, p. 50.

7. Pour davantage de précisions, on peut se reporter à l'ouvrage d'Ellis WATERHOUSE, Painting in Britain 1530-1790, New Haven: Yale University Press, 1994. Voir le chapitre 13 sur les 'conversation pieces and fancy pictures'.

8. John CORNFORTH, Early Georgian Interiors, Yale University Press, 2004, p. 191.

9. Yale Center for British Art, Paul Mellon Collection

10. Royal Collection.

11. Philadelphia Museum of Art: John H. McFadden Collection.

12. Newhouse Galleries, New York.

13. John CORNFORTH analyse la décoration de Wentworth Castle dans Early Georgian Interiors, New Haven: Yale University Press, 2004, p.35.

14. Tate Gallery, Londres. <http://www.tate.org.uk/servlet/ViewWork?workid=6614>

15. Bridgeman Art Library

16. National Portrait Gallery, Londres. <http://www.npg.org.uk/live/search/portrait.asp? search=ss\&sText=Jonathan + Tyers $+\&$ LinkID $=$ mp05854\&rNo=0\&role=sit $>$

17. Huntington Library, Pasadena.

18. Collection privée.

19. David H. SOLKIN fait le parallèle entre les peintures de la cérémonie du thé dans la première et la deuxième moitié du dix-huitième siècle dans Painting for Money: The Visual Arts and the Public Sphere in Eighteenth-Century England, New Haven : Yale University Press, 1993, p. 105.

20. Yale Center for British Art : Paul Mellon Collection.

21. Getty Museum. Visible sur: <http://rubens.anu.edu.au/htdocs/surveys/ charlotte/0321/032156.JPG>

22. Collection of Messrs Ehrich Brothers.

23. Tate Gallery, Londres. Visible sur: <http://www.tate.org.uk/servlet/ViewWork? workid $=10300 \&$ searchid $=35751>$

24. Visible sur : <http://www.pemberley.com/janeinfo/1814adwm.gif>

25. David HUME souligne les bienfaits du commerce dans son essai 'Of commerce', publié dans Essays Moral, Political and Literary, 1741-42 (Oxford: University Press, 1993, p. 156, p. 157, p. 163). Il écrit ainsi 'Foreign trade has preceded any refinement in home manufactures, and given birth to domestic luxury'.

26. National Gallery, Londres.

27. Musée Nissim de Camondo, Paris.

28. Musée national des châteaux de Versailles et de Trianon. 


\section{RÉSUMÉS}

$\mathrm{Au}$ dix-huitième siècle, un nouveau genre pictural apparaît en Angleterre avec l'essor du commerce du thé et l'évolution des modes de vie. La cérémonie du thé se développe parmi les classes dirigeantes et chez les négociants de l'East India Company, qui se font peindre dans des conversation pieces. Ces tableaux qui regroupent la famille et parfois les amis soulignent le statut social des personnages représentés dans leur environnement et révèlent les transformations des relations familiales. Ce genre national évolue avec les sensibilités et sera même tourné en dérision par quelques caricatures à la fin du siècle.

A new genre appeared in eighteenth-century Britain with the development of the tea trade and changing lifestyles. The tea party was widespread among the ruling classes and the traders of the East India Company who were painted in conversation pieces. These paintings, which brought together families and sometimes friends, focus on the social status of the characters shown in their environment and highlight the changes in family life. This national genre evolved with sensibilities and was even ridiculed by some caricatures at the end of the century.

\section{AUTEUR}

\section{SOPHIE LOUSSOUARN}

Université de Picardie Jules Verne (Amiens) 